\title{
Genomic consequences of apple improvement
}

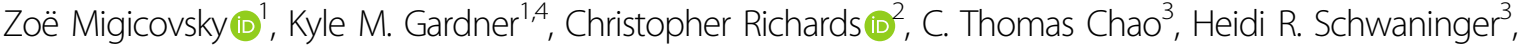 \\ Gennaro Fazio ${ }^{3}$, Gan-Yuan Zhong $\mathbb{1}^{3}$ and Sean Myles $^{1}$
}

\begin{abstract}
The apple (Malus domestica) is one of the world's most commercially important perennial crops and its improvement has been the focus of human effort for thousands of years. Here, we genetically characterise over 1000 apple accessions from the United States Department of Agriculture (USDA) germplasm collection using over 30,000 singlenucleotide polymorphisms (SNPs). We confirm the close genetic relationship between modern apple cultivars and their primary progenitor species, Malus sieversii from Central Asia, and find that cider apples derive more of their ancestry from the European crabapple, Malus sylvestris, than do dessert apples. We determine that most of the USDA collection is a large complex pedigree: over half of the collection is interconnected by a series of first-degree relationships. In addition, 15\% of the accessions have a first-degree relationship with one of the top 8 cultivars produced in the USA. With the exception of 'Honeycrisp', the top 8 cultivars are interconnected to each other via pedigree relationships. The cultivars 'Golden Delicious' and 'Red Delicious' were found to have over 60 first-degree relatives, consistent with their repeated use by apple breeders. We detected a signature of intense selection for red skin and provide evidence that breeders also selected for increased firmness. Our results suggest that Americans are eating apples largely from a single family tree and that the apple's future improvement will benefit from increased exploitation of its tremendous natural genetic diversity.
\end{abstract}

\section{Introduction}

Plant domestication is the artificial selection for phenotypic changes. These changes are often maladaptive to the organism in the wild, but of benefit to humans, leading to genetic differentiation between the wild progenitor species and contemporary commercial cultivars. In many species, domestication involves an intense population bottleneck, which reduces genetic variation. In contrast, long-lived perennial species, including fruit trees, generally experienced mild domestication bottlenecks owing to several factors including the extensive use of clonal propagation, long juvenile phases, and predominantly outcrossing mating habit ${ }^{1}$.

Correspondence: Gan-Yuan Zhong (GanYuan.Zhong@ars.usda.gov) or Sean Myles (sean.myles@dal.ca)

1 Department of Plant, Food, and Environmental Sciences,

Faculty of Agriculture, Dalhousie University, Truro, NS, Canada

${ }^{2}$ USDA-ARS, Plant Germplasm Preservation Research Unit, Fort Collins, CO, USA

Full list of author information is available at the end of the article
The domesticated apple (Malus $\mathrm{x}$ domestica Borkh.), is one of the most economically important fruit crops, with a worldwide production value exceeded only by tomatoes (Solanum lycopersicum L.) and grapes (Vitis vinifera L.) ${ }^{2}$. In the first half of the twentieth century, Nikolai Vavilov suggested that the origin of apple domestication coincided with the centre of phenotypic diversity of the presumed ancestral species Malus sieversii (Ledeb.) Roem., located in the Tien Shan mountains along Kazakhstan's eastern border with $\mathrm{China}^{3}$. This speculation was largely supported by recent analyses of genetic data, which identified $M$. sieversii as the primary progenitor species for $M$. domestica $^{4}$. In addition, over $20 \%$ of the $M$. domestica genome was likely derived from the wild European crabapple Malus sylvestris (L.) Mill ${ }^{5,6}$.

Plant domestication can be understood as a continuum rather than a single event, and selection continues over time as plant breeders target traits for improvement. While there is an immense amount of genetic variation available in apple due to a weak domestication 
bottleneck $^{6,7}$, most commercial production focuses on a limited number of elite cultivars. In 2017 , over $50 \%$ of the commercial apple production in the European Union consisted of only five apple cultivars ${ }^{8}$. The repeated use of a small number of elite cultivars during breeding reduces genetic diversity, especially among commercial cultivars. In addition, clonal propagation allows successful cultivars to persist for long periods of time, such as the 'McIntosh' apple, which is still in widespread production after 200 years $^{9,10}$. The ability of the apple industry to respond to pests, pathogens, and a changing climate will rely on comprehensive evaluations of apple variation, and the subsequent introgression of desirable genetic variants into apple breeding material.

In this study, we investigated (1) the domestication history of $M$. domestica by examining its relationship to the progenitor species $M$. sieversii and M. sylvestris, (2) signatures of positive selection during domestication and improvement, and (3) the improvement history of $M$. domestica by examining the relationships among modern cultivars in the USDA apple germplasm collection.

\section{Materials and methods}

\section{Genotype data collection}

The apples (Malus spp.) investigated here are from the USDA apple germplasm repository in Geneva, NY, USA. Leaf tissue was collected from 1949 accessions. The countries of origin of the accessions are indicated in Supplementary Fig. S1 and Supplementary Table S1. DNA was extracted from these accessions using commercial extraction kits. Genotyping-by-sequencing (GBS) libraries were generated according to Elshire et al. ${ }^{11}$. A visual overview of all data processing and analysis steps described below is provided in Supplementary Fig. S2.

The samples were processed with two different restriction enzymes (ApeKI, PstI/EcoT22I) in separate GBS libraries and were sequenced using Illumina Hi-Seq 2000 technology (96 samples per lane) at Cornell University (Ithaca, New York, US) across 42 lanes generating 100-bp single-end reads. The DNA sequence data are as NCBI BioProject PRJNA636391. Reads that failed Illumina's 'chastity filter' were removed and remaining reads were aligned to the Malus x domestica GDDH13 v1.1 reference genome ${ }^{12}$ using Burrows-Wheeler aligner tool $\mathrm{v} 0.7 .12^{13}$ and the Tassel version 5 pipeline ${ }^{14}$. Kmerlength was set to 82 for ApeKI and 89 for PstI-EcoT22I, and the minMAF was set to 0.01 during the DiscoverySNPCallerPluginV2 step. Non-biallelic sites and indels were removed using VCFtools v0.1.14 ${ }^{15}$. VCF files for both enzymes were then merged using a custom Perl script that preferentially kept SNPs called from the PstI-EcoT22I libraries in cases where SNPs were identified from both restriction enzymes. The resulting data set contained 1949 accessions and 1,103,605 SNPs. Mean read depth per individual, per SNP, and the proportion of heterozygotes per site were calculated using VCFtools v0.1.14 ${ }^{15}$ (Supplementary Fig. S3).

Missing genotypes in the VCF files were imputed using LinkImpute $\mathrm{R}^{16}$ with the following filters: max missingness of 0.30 , minor allele frequency (MAF) of 0.01 , minimum depth of 8 , and Hardy-Weinberg equilibrium threshold of $p=0.0001$. The resulting data set had an imputation accuracy of 0.9778 and a correlation value of 0.8764 , with 1598 accessions and 68,392 SNPs remaining.

The data set was filtered to only include accessions in the USDA apple germplasm collection that were relevant to modern apple development, which includes accessions labelled as $M$. domestica $(N=1154), M$. sieversii $(N=$ 195), Malus (L.) baccata Borkh. $(N=40)$, Malus floribunda Sieb. ex Van Houtte $(N=17)$, Malus orientalis Uglitzk. $(N=17)$, and $M$. sylvestris $(N=15)$. Next, the VCF file was converted using PLINK v1.07 ${ }^{17,18}$ and filtered for MAF 0.01, resulting in 1438 accessions and 47,925 SNPs.

Our genotype calling pipeline assumes all accessions are diploid (2x), and we, therefore, aimed to exclude triploid accessions (3x). Previous work has confirmed that triploids can be identified from GBS data due to their excessive heterozygosity ${ }^{19}$. We examined heterozygosity by individual, and contrasted these values with labels available in the USDA germplasm database for $2 x, 3 x$ and $4 \mathrm{x}$ accessions (Supplementary Fig. S4). Using a Tukey test, we determined that accessions labelled as $3 \mathrm{x}$ were significantly more heterozygous than $2 \mathrm{x}\left(p<1 \times 10^{-15}\right)$ or $4 \mathrm{x}$ $\left(p=1.385 \times 10^{-4}\right)$ individuals. There was no significant difference in heterozygosity between $2 \mathrm{x}$ and $4 \mathrm{x}$ accessions, indicating that the accessions labelled as $4 \mathrm{x}$ were likely all autotetraploids and could, therefore, be treated as diploid for the purposes of genotype calling and all downstream analyses. The mean proportion of heterozygous genotypes was 0.191 for accessions labelled as $2 \mathrm{x}, 0.226$ for $3 \mathrm{x}$ accessions, and 0.182 for $4 \mathrm{x}$ accessions. Based on these results we removed 168 accessions with heterozygosity $>0.21$ that we inferred to be triploid, including 28 labelled as $2 x, 51$ labelled as $3 x$, and 2 labelled as $4 x$. There were 62 accessions labelled as $3 x$ that were not removed using this filter. The majority of the accessions removed $(N=$ 147) were labelled as M. domestica. After filtering, 1270 accessions remained.

\section{Sample curation}

To address potential mislabelling of species within the USDA apple germplasm collection, we used the programme fastSTRUCTURE ${ }^{20}$ to evaluate relatedness among samples. First, we included only SNPs anchored to chromosomes 1 to 17 , resulting in 46,022 SNPs. Next, we removed accessions that were suspected of being clonally related. To do this, we calculated pairwise identity-by-descent (IBD) using PLINK $^{17,18}$ and only retained one accession per clonal 
group where proportion IBD (pi-hat) $>0.9$, which removed a further 207 accessions. Only SNPs with MAF $>0.01$ in the remaining accessions were retained, and these were then pruned for linkage disequilibrium (LD) using the PLINK filter (--indep-pairwise 103 0.5), resulting in 27,871 SNPs. The data set of 1063 accessions included samples labelled as $M$. domestica $(N=822), M$. sieversii $(N=184), M$. baccata $(N=25), M$. floribunda $(N=2), M$. orientalis $(N=17)$, and $M$. sylvestris $(N=13)$. We examined values of $K$ from 3 to 10 using fastSTRUCTURE and decided on $K=3$ based on the choose.py function, which determined that three components were needed to explain structure in the data (Supplementary Fig. S5). $K=3$ resulted in a partition containing primarily $M$. domestica, a partition containing primarily $M$. sieversii, and a partition containing primarily $M$. floribunda and M. baccata accessions.

Our aim was to assess the process of domestication and breeding before the use of these wild species during modern cultivar development, and thus, we excluded samples that are likely recent hybrids generated from the use of these wild species during modern breeding. Some samples labelled as $M$. sieversii were suspected to be the result of hybridisation with $M$. domestica cultivars, and we also aimed to exclude these from downstream analyses. To achieve this, we removed from further analysis $67 \mathrm{M}$. domestica accessions with $<0.80$ assignment probability to the $M$. domestica cluster and $69 M$. sieversii accessions with $<0.80$ identity to the $M$. sieversii cluster.

\section{Relationship between domesticated apples and their progenitors}

We returned to the genotype table prior to MAF and LD filtering for fastSTRUCTURE analysis and retained only accessions identified as primarily $M$. domestica, $M$. sieversii, and $M$. sylvestris. Next, we repeated the MAF filter of 0.01 and LD-pruning using PLINK. The curated Malus data set contained 883 accessions and 23,006 SNPs.

A principal components analysis (PCA) was conducted with the smartPCA module of the EIGENSOFT package $^{21}$. Equal sample sizes of the ancestral populations ( $N=13$ for $M$. sieversii and $M$. sylvestris) were selected as this has been shown to be a crucial factor in accurately inferring genetic relatedness based on $\mathrm{PCA}^{22}$. After establishing the PC axes based on these ancestral populations, the remaining $M$. sieversii and $M$. domestica accessions were projected onto the axes and 13 accessions were identified that were likely mislabelled or hybrids, and these were either re-labelled or removed (Supplementary Fig. S6). We repeated the PCA excluding the mislabelled samples, which included $11 \mathrm{M}$. sylvestris, $115 \mathrm{M}$. sieversii and $749 \mathrm{M}$. domestica and a total of 22,934 SNPs after a MAF filter of 0.01 and LD-pruning as described above. We established PC axes using 11 accessions for $M$. sieversii and $M$. sylvestris and then projected the remaining accessions onto these axes as described previously $^{23,24}$.

In order to detect if gene flow between wild apples $(M$. sieversii and $M$. sylvestris) and domesticated apples differed for cider and dessert apples, we labelled accessions according to primary use based on Migicovsky et al. ${ }^{25}$. We used the label 'dessert' to include cultivars labelled as both dessert and cooking apples in the USDA Germplasm Resources Information Network (GRIN) database and other online sources. A Mann-Whitney $U$-test was performed to determine if cider $(N=69)$ and dessert $(N=$ 288) apples significantly differed along PC1 and PC2. Finally, we computed F3 statistics using the qp3Pop test in ADMIXTOOLS ${ }^{26}$ to test for introgression from wild species into cider or dessert apples.

\section{Genome-wide scan for selection in $M$. domestica}

To identify genomic regions that may have been targets of positive selection in $M$. domestica, we compared $M$. domestica $(N=749)$ to $M$. sieversii $(N=115)$ using 33,266 SNPs. Genomic regions under positive selection since domestication in $M$. domestica should be significantly differentiated from its primary progenitor, $M$. sieversii. We calculated Fst for each SNP according to Weir and Cockerham ${ }^{27}$ and considered SNPs with Fst values within the top 5\% of genome-wide Fst values as potential selection candidates.

Next, we inferred haplotypes using fastPHASE ${ }^{28}$ and calculated the cross population extended haplotype homozgosity (XP-EHH) statistic using the selscan software with the --trunc-ok and --max-gap 1,500,000 options $^{29}$. We contrasted haplotype diversity between $M$. sieversii (the 'reference' population) and $M$. domestica. Similar to Fst, we considered selection candidates as those SNPs with XP-EHH values within the top $5 \%$ of positive values genome-wide, indicating extreme haplotype homozygosity in $M$. domestica. Genomic regions containing SNPs within the top 5\% of both the Fst and the $\mathrm{XP}-\mathrm{EHH}$ distributions were identified as putatively under selection in M. domestica.

Within the genomic regions under putative selection during domestication we conducted a gene ontology enrichment analysis to determine if any GO terms were overrepresented, suggesting possible metabolic or functional changes in the apple genome due to domestication. To conduct the analysis, we obtained the complete gene annotation data set from the Genome Database for Rosaceae that accompanied version GDDH13 v1.1 of the reference genome ${ }^{12,30}$.

We searched for all genes where the entire region fell within $+/-50 \mathrm{~kb}$ of a SNP identified as a candidate for selection and reduced the list to unique genes (MD IDs), which resulted in 1738 genes of interest, including 1073 
which were associated with at least one GO term. We imported the genome-wide annotations as well as those under putative selection in $M$. domestica into the topGO package in $\mathrm{R}^{31}$. We used topGO to test for gene enrichment in biological process ontology using the algorithm $=$ 'weight01', which takes GO hierarchy into account, and the statistic $=$ 'fisher'.

\section{Relatedness within $M$. domestica}

To investigate patterns of relatedness within $M$. domestica, we filtered the genotype table, following removal of triploid accessions, for only accessions labelled as $M$. domestica, regardless of hybridisation. The resulting data set included $1005 \mathrm{M}$. domestica accessions and an additional MAF filter of 0.01 resulted in 31,426 SNPs remaining. We estimated IBD for all pairwise comparisons among the accessions using PLINK ${ }^{17,18}$. Groups of clones were defined as having IBD $>0.90$ among all members. We visualised clonal relationships using the 'network' package in $\mathrm{R}^{32}$.

Next, we reduced the data set to unique cultivars by only including one accession from each clonal group, resulting in the removal of 179 accessions. After filtering for MAF $>0.01$, the final data set contained 31,378 SNPs genotyped in 826 accessions. We used the pairwise IBD matrix to identify putative first-degree relationships (i.e., parent-offspring, full sibling, or equivalent) among the $M$. domestica samples. The expected IBD value for firstdegree relationships is 0.5 . However, observed IBD values for first-degree relatives are expected to vary due to genotyping error, errors in reference genome assembly, and low and uneven SNP density. To calibrate our IBD thresholds for defining first-degree relatives, we identified 55 known parent-offspring pairs from the literature and examined the range of IBD values (Supplementary Table $\mathrm{S} 2)^{33,34}$. We removed the two lowest values, which were likely due to mislabelled accessions. The remaining IBD values ranged from 0.4158 to 0.5625 , and we therefore considered pairs of samples with IBD values greater than or equal to 0.4158 and less than or equal to 0.5625 as putative first-degree relatives. As a result, accessions referred to as first-degree relatives within this manuscript are inferred based on IBD values. Such values do not necessarily reflect first-degree relatives and could be generated from more complex familial relationships due to backcrossing to close relatives and other complex crossing schemes across generations. In addition, some accessions, which represent true first-degree relatives may not fall within this range of IBD values and, therefore, be missed by this analysis. We visualised first-degree relatives using the 'network' package in $\mathrm{R}^{32}$. Lastly, we reduced the data set to only accessions which had a first-degree relationship with one of the top 9 apple cultivars sold in the USA in $2018^{10}$. Sequencing for one of the cultivars,
'Empire', failed and therefore our analyses were restricted to 8 of the top 9 cultivars, which included 'Gala', 'Red Delicious', 'Granny Smith', 'Fuji', 'Honeycrisp', 'Golden Delicious', 'McIntosh', and 'Pink Lady®'. Visualisations of pedigree relationships were produced using the 'network' package in $\mathrm{R}^{32}$.

\section{Genome-wide scan for selection in $M$. domestica during recent breeding}

Within $M$. domestica, we examined dessert and cider apples for evidence of selection using both Fst and XPEHH scans, as performed above for $M$. domestica and $M$. sieversii. SNPs in the top 5\% of Fst values were separately tested for overlap with the highest (selection in dessert apples) or lowest (selection in cider apples) 5\% of XP$\mathrm{EHH}$ values. Once again, we tested unique genes within $+/-50 \mathrm{~kb}$ of a SNP for GO enrichment using the topGO package in $\mathrm{R}^{31}$.

Next, we conducted genome-wide association study (GWAS) and XP-EHH scans for traits that may have experienced improvement after domestication. A new reference genome ${ }^{12}$ and new imputation method ${ }^{16}$ were made available following the publication of our previous work and, therefore, our current study includes over three times as many SNPs as the initial GWAS performed in Migicovsky et al. ${ }^{25}$. We therefore retrieved the phenotype data from Migicovsky et al. ${ }^{25}$ and repeated the GWAS for fruit colour (red $(N=389)$ or green $(N=131)$ ), fruit firmness (soft $(N=278)$ or firm $(N=310))$ and fruit size (small $(N=320)$ or large $(N=276))$ using Tassel v.5.2.48 with the inclusion of a kinship matrix and PCs 1 to $3^{35}$. For each of these binary phenotypes, we performed an $\mathrm{XP}-\mathrm{EHH}$ analysis by comparing accessions with one phenotype (e.g., red) to accessions with the other (e.g., green). To verify that the colour GWAS identified only a single significant locus, we used a multi-locus model in the R package 'mlmm' v.0.1.1 to perform GWAS $^{36}$ again including the kinship matrix and PCs 1 to 3. MLMM incorporates significant SNPs as cofactors using a stepwise regression. The optimal model was selected using the extended Bayesian information criterion (EBIC) and plotted using the plot_opt_GWAS function ${ }^{36}$. We examined the overlap between the top 5\% of XP-EHH SNPs and those passing the Bonferroni-corrected threshold for GWAS. GWAS and XP-EHH results were visualised using the 'qqman' $\mathrm{R}$ package ${ }^{37}$, with the location of the D5Y SNP (chr3:30698039) in NAC18.1 (MD03G1222600) indicated on the firmness plot.

The eight cultivars we sequenced that are among the top apples sold in the USA were all homozygous for the desirable firm Y allele at the D5Y SNP in the NAC18.1 gene. To determine the likelihood of observing homozygosity for this allele across eight random cultivars, we randomly sampled $8 \mathrm{M}$. domestica accessions without replacement 
10,000 times and counted the frequency with which all eight accessions were homozygous for the firm allele.

\section{Results}

We collected over 780 billion nucleotides of DNA sequence from the USDA apple germplasm collection, which includes 1949 apple accessions originating from 50 different countries. We used these data to correct and improve the labelling of accessions, and to remove samples unsuitable for downstream analyses. For example, we determined that approximately $13 \%$ of the $M$. domestica accessions within the collection were triploid (Supplementary Fig. S4). We also identified numerous instances where accessions were likely the result of recent hybridisation with wild species and/or accessions' species labels were incorrect (Supplementary Figs. S5 and S6). We implemented several quality filtering steps to ensure that each accession was assigned to the correct species before proceeding with downstream analyses, and each step is described in the Materials and Methods, Table S1 and Supplementary Fig. S2.

\section{Genomic insights into apple domestication}

To examine the effects of domestication on apple genetic diversity, we assessed the genetic contributions of the wild relatives, M. sieversii and M. sylvestris, to cider and dessert apples. Using the F3 test, cider ( $\mathrm{f} 3=-0.055173)$ and dessert apples $(\mathrm{f} 3=-0.042006)$ had negative $\mathrm{f} 3$ values, suggesting that both cider and dessert apples included ancestry from both wild relatives, $M$. sieversii and M. sylvestris.
We investigated the relative contribution of each wild ancestor to cider and dessert apples by performing ancestry deconvolution using PCA. Our analysis identified two distinct groups of $M$. sylvestris along PC2: accessions from Germany $(\mathrm{PC} 2>0)$ and Macedonia $(\mathrm{PC} 2<0)$. By projecting both cider and dessert apples onto the PCs, we found that cider apples were significantly differentiated from dessert apples along PC1 $\left(W=14922, p=9.439 \times 10^{-11}\right)$, which indicates that cider apples derive more ancestry from $M$. sylvestris while dessert apples derive more ancestry from $M$. sieversii (Fig. 1). Cider apples were also significantly different from dessert apples along PC2 ( $W=14582, p=1.598 \times$ $\left.10^{-9}\right)$, with cider apples appearing more closely related to German M. sylvestris accessions compared to accessions from Macedonia (Supplementary Fig. S7).

To identify regions of the genome that may have experienced selection during domestication, we compared the genomes of $M$. domestica to the genomes of its primary progenitor species, $M$. sieversii, using Fst (Supplementary Table S3 and Fig. 2A) and XP-EHH (Supplementary Table S4 and Fig. 2B). There were 265 SNPs with values within the top 5\% of both test statistics (Supplementary Table S5) and we tested for $\mathrm{GO}$ enrichment of the genes found within $50 \mathrm{~kb}$ of these SNPs (Supplementary Table S6). We report the top ten GO terms for enrichment (Supplementary Table S7), which included ion transport, lipid transport and positive regulation of kinase activity.

\section{Relatedness among apple cultivars}

By calculating IBD among all pairs of $1005 \mathrm{M}$. domestica accessions, we identified 641 pairs of accessions sharing a


Fig. 1 Principal components analysis (PCA) showing population structure for $M$. domestica $(N=749), M$. sieversii $(N=115)$, and $M$. sylvestris $(\boldsymbol{N}=\mathbf{1 1})$. A PC axes were established using equal sample sizes $(N=11)$ of the wild species and the remaining accessions were projected onto the axes. The percent variance explained by each PC is indicated in parentheses. B Comparison of PC1 values for cider and dessert apples demonstrates that dessert apples derive more ancestry from $M$. sieversii, while cider apples derive more ancestry from $M$. sylvestris 


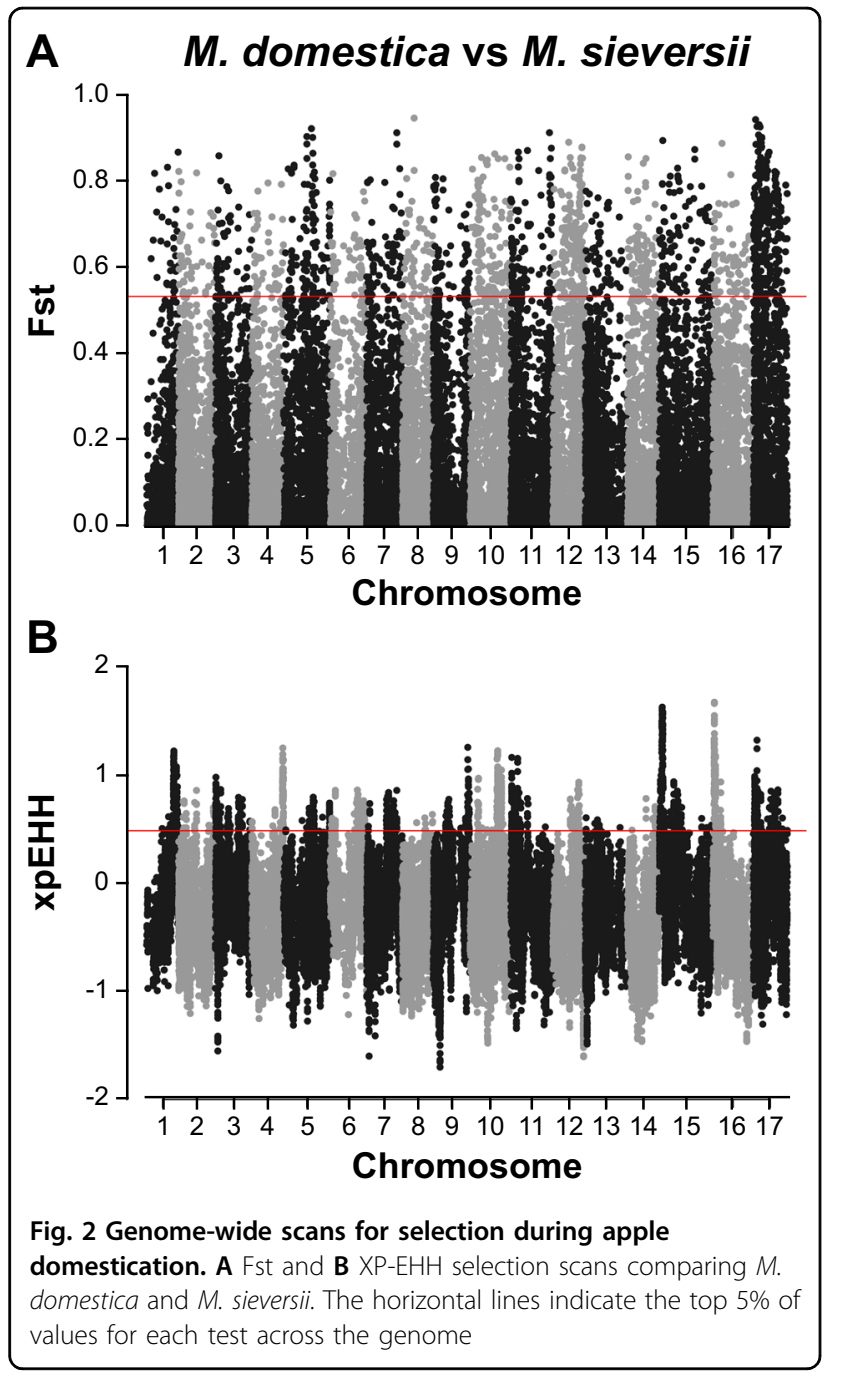

clonal relationship. In total, 279 accessions were distributed across 100 clonal groups, with the number of clonal relationships within each group ranging from 1 to 22 (Supplementary Table S8 and Fig. 3A, B). The accessions with the largest number of clonal relationships included 'Golden Delicious' (22), 'Red Delicious' (15), 'McIntosh' (10), and 'Northern Spy' (8).

After retaining only one accession from each clonal group, we determined that 535 of the remaining 826 unique accessions had at least one first-degree relative (i.e., sibling, parent-offspring relationship, or equivalent) with another accession in the collection. 'Golden Delicious' had the largest number of first-degree relatives (66), while 'Red Delicious' had the second largest number of relatives (61). Most accessions (314) had 1 or 2 firstdegree relatives, while 44 accessions had $>10$ (Supplementary Table S9). Over half of the collection (435 out of 826 accessions) were interconnected by a series of firstdegree relationships and thus belonged to a single extended pedigree (Fig. 3C, D). When we restricted our analysis to relatives of the top 8 apple cultivars sold in the United States, we identified 129 accessions with a firstdegree relationship with at least one of the top 8 apple cultivars (Fig. 4).

\section{Selection during apple improvement}

We aimed to identify regions of the apple genome that experienced positive selection due to breeding and improvement. First, we compared dessert and cider apples to identify regions putatively under selection using both Fst (Fig. 5A and Supplementary Table S10) and XP-EHH (Fig. 5B and Supplementary Table S11). Based on the overlap across these two scans, there were 433 SNPs identified as putatively under selection in dessert apples and 81 SNPs in cider apples (Supplementary Table S12). In both instances, we tested for $\mathrm{GO}$ enrichment of genes found within $50 \mathrm{~kb}$ of these SNPs (Supplementary Table S13) and reported the top ten GO terms (Supplementary Table S14). For dessert apples, the top term was flavonoid biosynthesis while for cider apples, the top terms included malate transport.

In addition to the comparison between dessert and cider apples, we conducted GWAS in M. domestica accessions for traits likely targeted by breeders and determined if SNPs significantly associated with these traits overlapped with signatures of recent selection as measured by the XPEHH statistic. We identified a GWAS signal when comparing red vs green apples on chromosome 9 at the $M Y B 1$ gene responsible for fruit colour ${ }^{38-42}$ (Fig. 6A) and found a strong signature of selection at this locus (Fig. 6B). The GWAS resulted in 31 significant SNPs, 22 of which also exhibited extreme positive values of the XP-EHH statistic, indicating intense selection for red skinned apples (Supplementary Table S15). The SNPs with selection signals extended over a $3.43 \mathrm{Mb}$ region on chromosome 9 (chr9:32436474-35868403) and overlapped with the MYB1 gene (MD09G1278600, chr9:35542733-35549175). We verified that our GWAS signal captures only a single locus of large effect on chromosome 9 and that the SNPs exceeding the significance threshold on other chromosomes were likely mismapped in the reference genome (Supplementary Fig. S8).

Following the same procedure, we tested for selection for increased fruit size. We failed to detect significant SNPs in our GWAS for apple size and did not find any convincing regions of selection for fruit size among SNPs with extreme XP-EHH values (Supplementary Table S16 and Supplementary Fig. S9). For example, the GWAS SNP with the lowest $p$-value on chromosome 13 was located over $350 \mathrm{~kb}$ away from a SNP with an extreme XP-EHH value. However, the GWAS SNP with the second lowest $p$-value (chr3:37279998) did exhibit an extreme XP-EHH value indicating a potential signature of selection for large fruit at this locus. This SNP falls within the LEA gene 

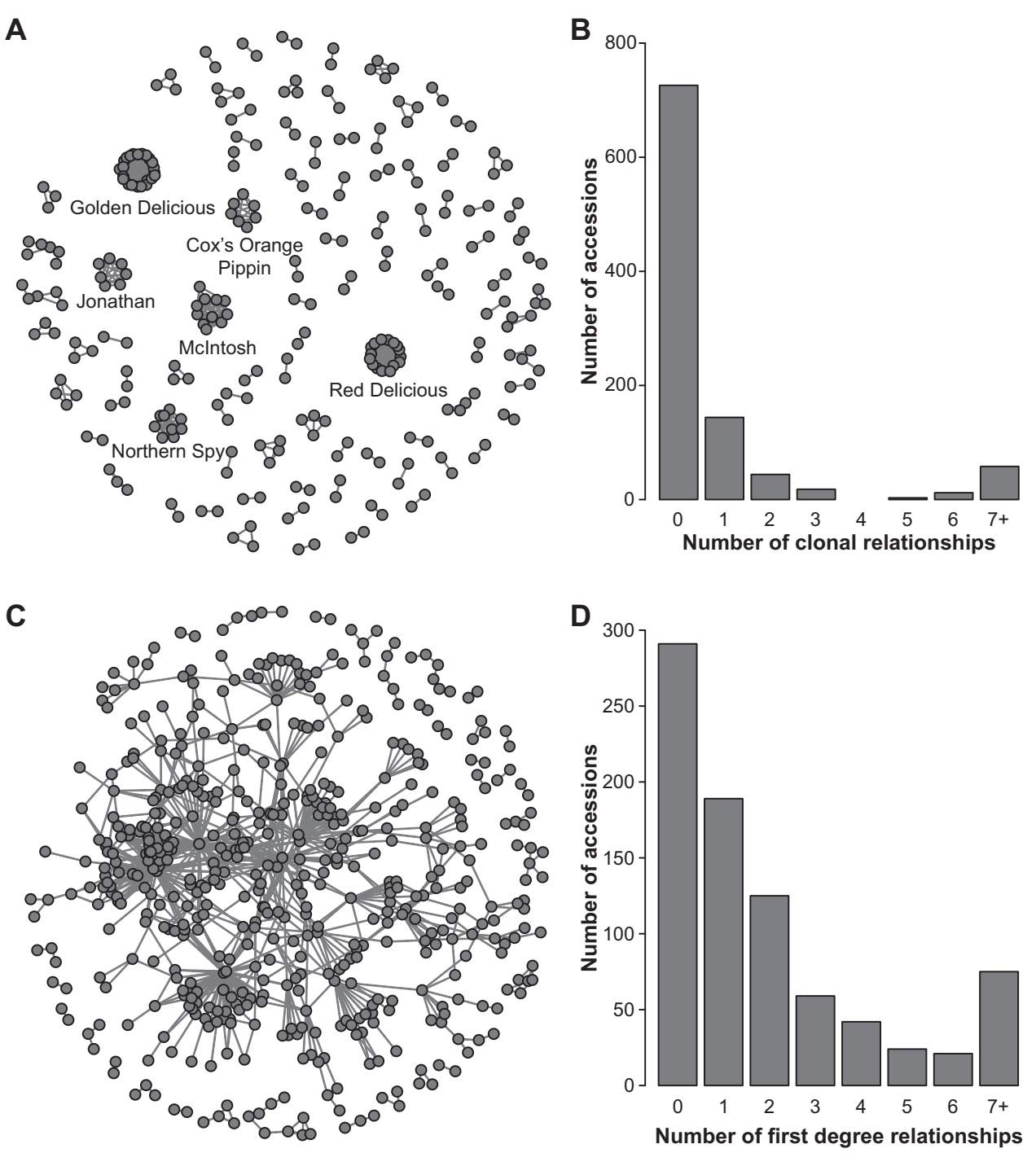

Fig. 3 Clonal and first-degree relationships within the USDA apple collection. A Network of clonal relationships among accessions labelled as M. domestica. Only accessions with at least one clonal relationship $(N=279)$ are included. Each accession is represented by a dot and each line represents a clonal relationship. B Number of clonal relationships across entire collection $(N=1005)$. C Network of first-degree relationships among apple accessions. Only accessions with at least one first-degree relationship $(N=535)$ are included. Each accession is represented by a dot and each line represents a first-degree relationship. D Number of first-degree relationships for each of the unique accessions within the collection $(N=826)$

(chr3:37278889-37280205; MD03G1296800), which encodes the late embryogenesis abundant (LEA) protein.

We compared soft and firm apples, but did not identify an overlap between the top 5\% of XP-EHH values and the region of the genome with the lowest $p$-values from the GWAS (Fig. 7). While no SNPs were found to be significant in the GWAS, the SNP with the lowest $p$-value is located at chr3:30698039 and falls within NAC18.1. This SNP, D5Y, results in a nonsynonymous substitution from aspartic acid (D) to tyrosine (Y) at the fifth amino acid of NAC18.1 and the Y allele has been associated with apple firmness and harvest time in previous studies ${ }^{25,43,44}$. While this putatively causal SNP was not among the 5\% most extreme XP-EHH values genome-wide, it was within the top $5.4 \%$ of values and thus nearly reached the $5 \%$ threshold employed here indicating possible selection for firmer apples (Supplementary Table S17).

We determined that $70.2 \%$ of $M$. domestica, $12.2 \%$ of $M$. sieversii, and $100 \%$ of $M$. sylvestris were homozygous for the firm Y allele. Among the remaining accessions, 23.5\% of $M$. domestica and $33.9 \%$ of $M$. sieversii were heterozygous, while all other accessions were homozygous for the soft D allele. All of the top 8 apples sold in the United States were homozygous for the desirable Y allele, and we determined that the probability of eight random accessions all being homozygous for the $\mathrm{Y}$ allele was $5.7 \%$. 


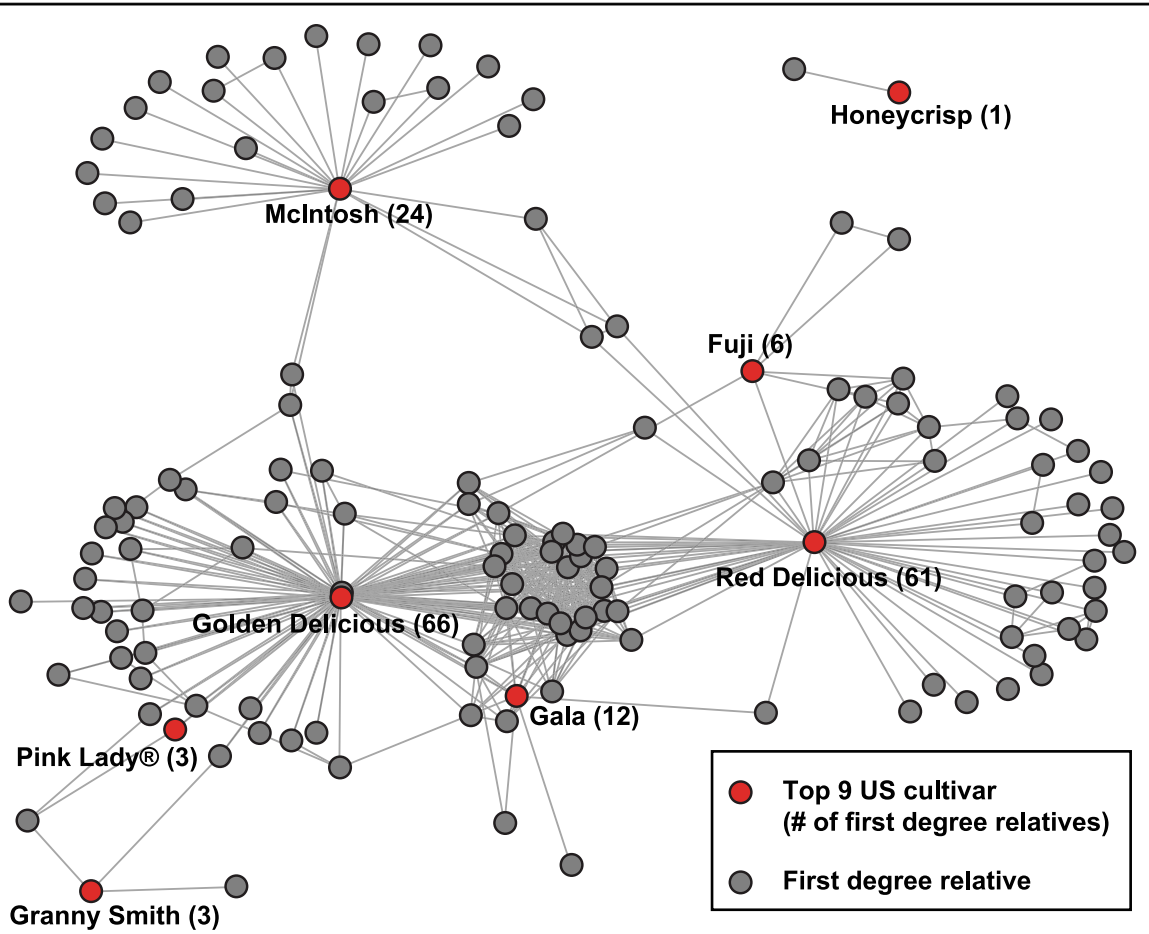

Fig. 4 Network of first-degree relationships with the top 8 apple cultivars sold in the United States. In addition to the top apple cultivars, only accessions with at least one first-degree relationship $(N=129)$ are included. Each accession is represented by a dot and each line represents a firstdegree relationship

\section{Discussion}

\section{Insights into apple domestication}

There is strong evidence that the apple was domesticated in the forests of Central Asia from the wild progenitor species, $M$. sieversii ${ }^{4,5,45}$. The degree to which there was subsequent hybridisation from other wild species during the several thousand years of its journey around the world remains a topic of intense investigation ${ }^{6,46-50}$. We determined that cider and dessert apples derive ancestry from both $M$. sieversii and the wild European crabapple, M. sylvestris. Genomic analyses found support for an Asian origin of the domesticated grape with subsequent introgression from wild species as the grape moved into Europe ${ }^{51}$. Similarly, our results suggest that, as the apple travelled west from its ancestral home in Central Asia, it hybridised with wild European apples. Wild European species have therefore played a crucial role in shaping the genomes of two of the world's most abundant fruit species.

Using a PCA-based ancestry analysis, we provide evidence that cider apples are generally more closely related to the European crabapple, $M$. sylvestris, than dessert apples are (Fig. 1). Therefore, the genomic contribution of European crabapples is greater for cider apples than for dessert apples. In our study, we also find that cider apples are more closely related to crabapples from Germany compared to those from Macedonia, suggesting that introgression was likely concentrated in northern regions of the European trade routes (Supplementary Fig. S7). Previous work has suggested that the introgression from M. sylvestris likely occurred after the cultivated apple was domesticated from $M$. sieversii ${ }^{5}$. Additional work will be required to identify the precise genomic regions that have been introgressed from $M$. sylvestris, and the extent to which $M$. sylvestris contributed to cider apples' tart and tannic characteristics.

In contrast to our work, Cornille et al. ${ }^{6}$ examined 299 cider and dessert apples, primarily of French origin, and found significantly higher introgression from $M$. sylvestris in dessert apples compared to cider apples. The discrepancy between our findings and those of Cornille et al. may be due to differences in genome coverage and/or sample origin. For example, Cornille et al. relied on 26 microsatellites from primarily French cider apples, while our study made use of $>20,000$ SNPs from cider apples from a larger geographic range including France (26), the United Kingdom (33), the United States (9), and Sweden (1). Thus, accessions within the USDA germplasm collection represent broader cider apple diversity, and this may explain the differences in our results.

We also compared $M$. domestica to $M$. sieversii to examine the genome for evidence of selection during domestication using Fst and XP-EHH (Fig. 2). We failed to identify specific candidate domestication genes, but we found that putatively selected genomic regions were 


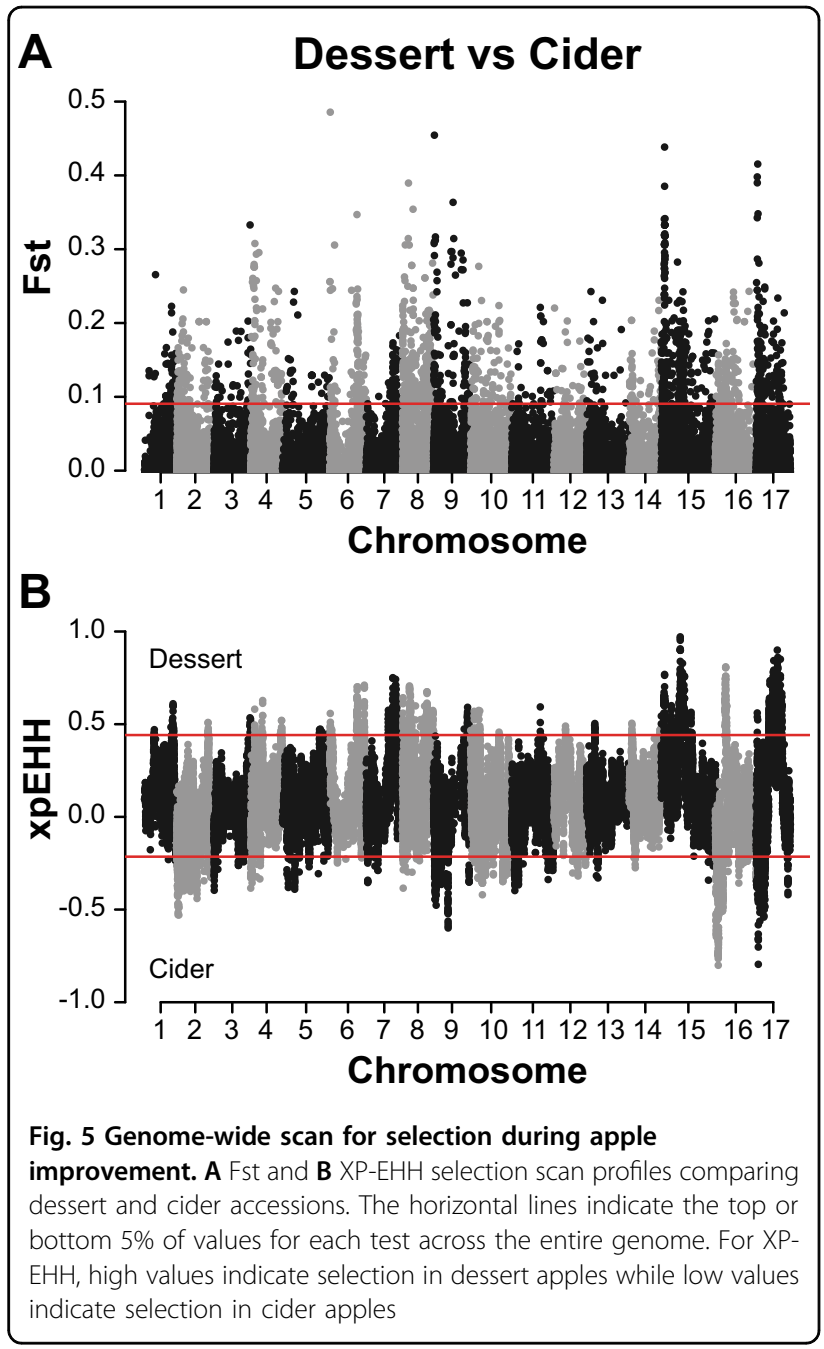

enriched for genes related to stress response, including target of rapamycin (TOR) signalling ${ }^{52}$, cellular response to phosphate starvation, and cellular response to stress. Recent work by Duan et al. ${ }^{5}$ reported that genes involved in abiotic stress were highly divergent between $M$. domestica and other wild species. This is consistent with our finding that genes involved in stress may have been selected for during apple domestication, potentially to enable adaptation to new environments.

\section{Relatedness among apple cultivars}

Apple is a clonally propagated crop and occasionally a 'somatic mutant' or 'sport' with a desirable phenotype arises, is clonally propagated, and subsequently commercialised. We found that the accessions with the highest number of sports, or clonal relationships, include many of the most widely sold cultivars in the USA such as 'Golden Delicious', 'Red Delicious', and 'McIntosh' (Fig. $3 \mathrm{~A})^{10}$. Among these is the 'Wijcik McIntosh', a 'McIntosh' sport with a columnar growth habit that makes it

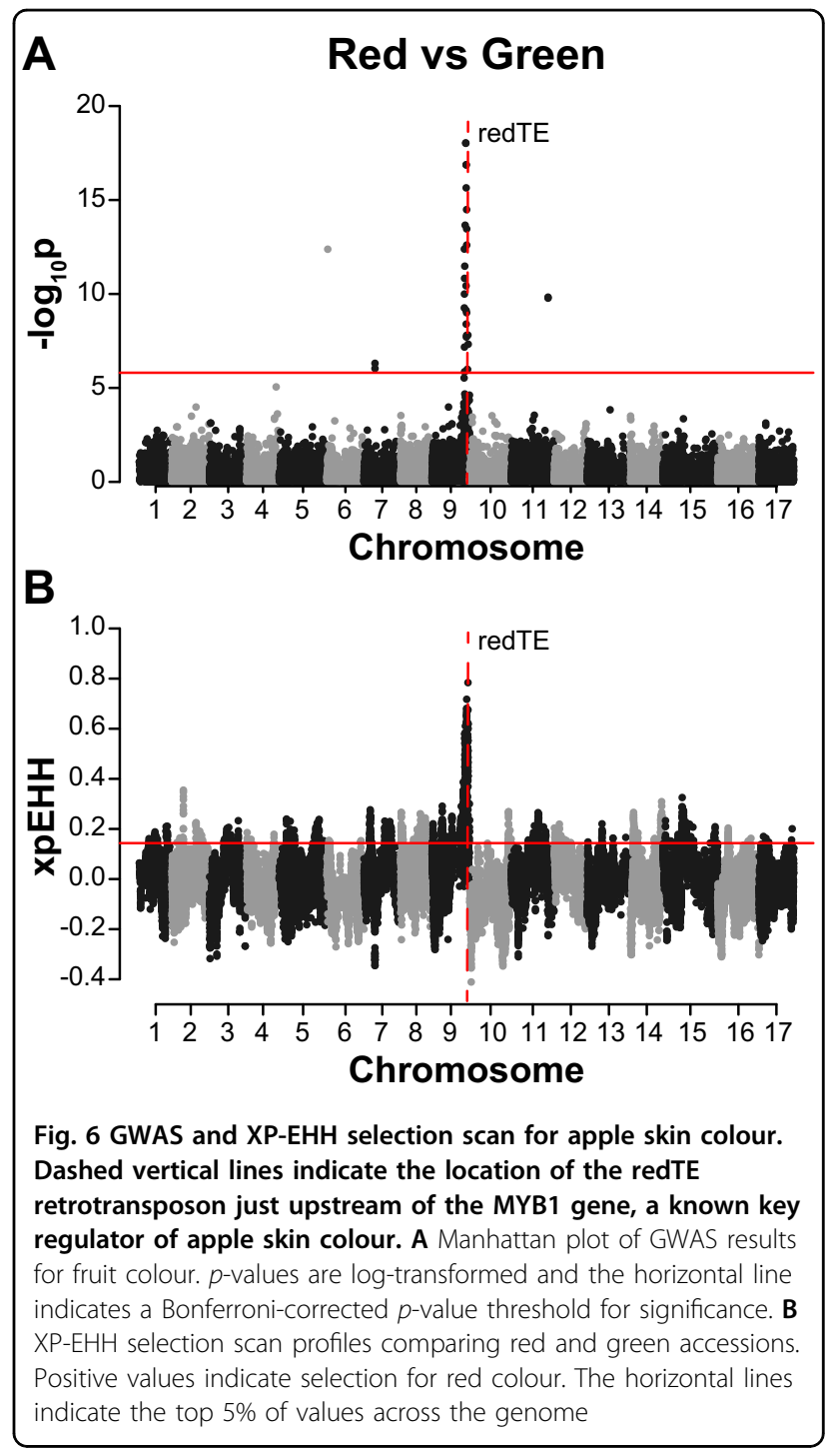

desirable for high-density planting ${ }^{53}$. However, the most common apple sports are those with redder fruit since red colour is commercially desirable and is a relatively easy trait to evaluate by eye ${ }^{54}$. For example, red sports of 'Fuji', like the accession 'Fuji Red Sport Type 2' that we genotyped, are more desirable than the original Fuji, which has poor red colouration ${ }^{55}$.

There are likely two explanations for the extensive clonal relatedness among elite apple cultivars. First, the probability of successful commercialisation of a new sport is likely highest when the desirable phenotype arises on the background of an already commercially successful cultivar. Second, successful cultivars are by definition the most widely planted, and since mutation is random, the probability of observing a sport is highest among the most abundant cultivars. A similar reasoning likely explains the large number of clones of the popular wine grapes 'Pinot Noir' and 'Chardonnay ${ }^{51,56}$. In total, over $27 \%$ of the 


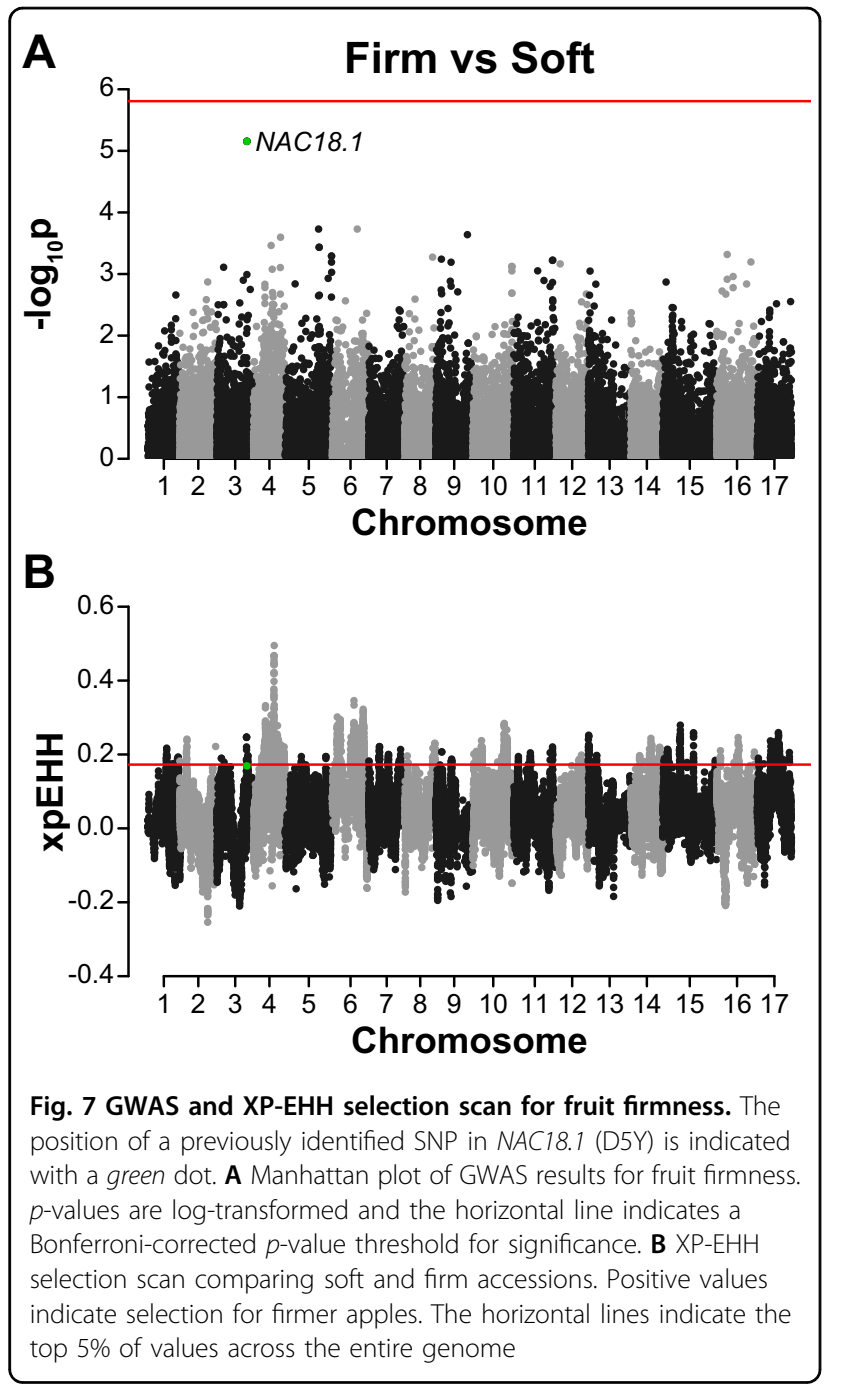

apples studied here had at least one clonal relationship (Fig. 3B), which suggests that breeders have frequently sought to improve cultivars by incorporating desirable phenotypes discovered initially as sports.

Historically, most of the world's commercially successful apple cultivars, like 'McIntosh', 'Granny Smith'57, 'Red Delicious', and 'Golden Delicious', were chance seedlings accidentally discovered from uncontrolled pollination events $^{54}$. However, most new cultivars, such as 'Pink Lady ${ }^{\circledR}$ ', 'Jazz ${ }^{\mathrm{TM}}$, and 'Honeycrisp' were developed by breeders who selected the most desirable trees from among the offspring of bi-parental crosses ${ }^{54}$. We provide evidence that the process of apple breeding involved the repeated use of a limited number of cultivars as parents, resulting in over half of the USDA apple germplasm collection being interconnected by a series of first-degree relationships (Fig. 3C, D). A genetic pedigree analysis of 1400 European apple accessions also revealed extensive relatedness by identifying over one thousand parent-offspring pairs ${ }^{58}$. Significant pedigree relatedness within a germplasm collection was also found in grapes, another clonally propagated perennial crop in which elite cultivars were repeatedly used during breeding ${ }^{51}$. In mango (Mangifera indica), most commercially important cultivars are the result of breeding in Florida, and many appear to be closely related to each other ${ }^{59}$. As was the case for clonal relationships, it is the apple cultivars 'Golden Delicious' and 'Red Delicious', which have the largest number of first-degree relatives: each has $>60$ firstdegree relatives in the collection. Notably, all of the top 8 apple cultivars grown in the USA, with the exception of 'Honeycrisp', were interconnected by a series of firstdegree relationships (Fig. 4). This means that Americans are eating apples largely from a single family tree.

While 'Honeycrisp' is not connected by a first-degree relationship to any of the other elite apple cultivars we genotyped, one of its recorded parents was 'Honeygold', an offspring of 'Golden Delicious', and the other was 'Macoun', an offspring of 'McIntosh' (Supplementary Table S9). Thus, presumably the breeder's intention in performing this cross was to use relatives of two of the most commercially successful apple cultivars in the USA as parents. However, it was discovered that Honeycrisp's recorded parentage was incorrect: the actual parents of 'Honeycrisp' are 'Keepsake', an offspring of 'Northern Spy', and MN1627, a University of Minnesota selection and offspring of 'Golden Delicious', which is no longer available and thus was not genotyped in our study ${ }^{60,61}$. Despite being derived from parents, which were not commercially successful, 'Honeycrisp' has achieved widespread commercial success and has been incredibly lucrative for apple growers ${ }^{10,61}$. Since its release in 1991, 'Honeycrisp' has been repeatedly used as a parent in breeding, including the release of at least eight commercial cultivars ${ }^{61}$. Perhaps the fact that neither of Honeycrisp's parents were commercially successful will motivate future apple breeders to explore the diversity that lies beyond the narrow relatedness network we identified here (Fig. 4).

Across the germplasm collection, over $15 \%$ of $M$. domestica cultivars had a first-degree relationship with at least one of the top 8 cultivars, indicating the recurrent use of a few cultivars in both breeding and commercial production. It takes about 25 years to develop and release a new apple cultivar ${ }^{54}$, so it is not surprising that apple breeders are likely to invest time and money into making crosses from 'tried and tested' cultivars. Using genomicsassisted breeding, however, there may be renewed interest in novel crosses-including the use of wild relativeswhen parents and offspring can easily be screened for desirable alleles ${ }^{62}$. From our results, it is clear that breeders have only just begun to tap into the tremendous diversity available in apple and its wild relatives. 


\section{Selection during apple improvement}

Among apples grown for commercial production, the primary use is fresh-eating (dessert) although cider apples are grown to be turned into juice and fermented into alcoholic cider. Cider apples are often referred to as 'spitters' because they are more tannic and acidic than dessert apples, which are desirable attributes when fermenting apple juice into cider. High conentrations of polyphenols, including flavonoids, increase astringency while malic acid contributes to tartness, both characteristics preferred for cider apples ${ }^{63,64}$. The genomic regions showing signatures of selection in dessert apples were enriched for genes involved in flavonoid biosynthesis, suggesting that there may have been selection in dessert apples for reduced polyphenol content. Signatures of selection in cider apples were identified in genomic regions enriched for genes involved in malate transport, which suggests that enhanced acidity may have been a primary target during cider apple improvement. Overall, these results suggest that genomic regions underlying astringency and tartness are highly differentiated between cider and dessert apples, and this differentiation likely underlies the primary differences in taste profile between these apple types.

In addition to variation in flavour, apples display a wide array of colours that contribute to consumer preference and that are controlled by both environmental and genetic factors. Apple skin colour has been shown to influence consumer preference with redder cultivars deemed to have better eating quality ${ }^{65}$. The long terminal repeat retrotransposon 'redTE', upstream of a MYB1 transcription factor, is a known key regulator of apple skin colour $^{42}$. A homologous $M Y B$ gene is also associated with fruit colour in date palms (Phoenix dactylifera) ${ }^{66}$ and Citrus $^{67}$. We identified a single locus associated with skin colour at MYB1 using GWAS (Fig. 6A and Supplementary Fig. S8). We also found a strong signature of positive selection at this locus, suggesting a rapid increase in the frequency of the 'redTE' allele due to selection for red skin during apple improvement (Fig. 6B). Our evidence of selection for a segregating mutation that improves apple redness is consistent with the observation that somatic mutations, leading to redder skin have also been selected for by breeders ${ }^{54}$. An extreme example of this is the 'Red Delicious' apple, which originated as a sport from the cultivar 'Hawkeye', a chance seedling found in 1872, which was supposedly less than $50 \%$ red $^{68}$. 'Hawkeye' was renamed 'Delicious', and in the nearly 150 years since its discovery, redder and redder sports, including 'Red Delicious', have been developed. While the original 'Delicious' is rarely grown, over 100 sports with improved colour are now available ${ }^{68,69}$. Our results provide strong genomic evidence that red skin was a key target during recent apple improvement.
In addition to selection for redder fruit, firmness is one of the top priorities for apple breeders ${ }^{70}$ and was likely a key target during apple improvement and breeding. In a study of pear (Pyrus spp.), 11 cell wall degradation-related genes were found in selective sweep regions, indicating possible selection for crisp fruit flesh in Asian pears ${ }^{71}$. In apple, the NAC18.1 gene has been associated with apple firmness in several recent studies ${ }^{25,43,72,73}$. While no SNPs were significantly associated with firmness in our GWAS, the D5Y mutation in NAC18.1 had the lowest $p$-value of all SNPs tested (Fig. 7). This suggests that we have detected a real association signal, but failed to achieve statistical significance because our GWAS was underpowered and/or our multiple testing correction was too strict. This same SNP was within the top 5.4\% of extreme $\mathrm{XP}$-EHH values genome-wide, which suggests that there may have been positive selection for apple firmness.

The weak signal of selection at NAC18.1 may be due to our observation that the desirable, 'firm' allele $(\mathrm{Y})$ is the ancestral allele among angiosperms ${ }^{25}$. However, the undesirable 'soft' allele (D) occurs at a much higher frequency within $M$. sieversii, the primary progenitor species of $M$. domestica. Over half (53.9\%) of the $M$. sieversii accessions were homozygous for the soft allele in comparison to only $6.28 \%$ of the $M$. domestica accessions. Thus, if there was selection for the ancestral firm allele, there may have been enough time for that allele to recombine onto numerous different haplotypes. XP-EHH is intended to detect extended homozygosity around a novel allele on a single haplotype that rapidly rises in frequency immediately after it appears in a population. This test is, therefore, underpowered to detect selection at NAC18.1, since the firm allele is ancestral and there have been very few generations of selection. It is worth noting, however, that all of the top 8 cultivars in the USA from the present study are homozygous for the firm allele, which is unlikely to be observed by chance given that the probability of homozygosity for this allele across eight random USDA accessions is $5.7 \%$. Altogether, our results suggest that, by selecting for firmness, apple breeders likely drove the firm NAC18.1 allele to high frequency.

\section{Conclusions}

Our genome-wide analysis of the USDA apple germplasm collection allowed us to determine the consequences of domestication and subsequent improvement on patterns of apple genetic diversity. By examining population structure among wild species and the domesticated apple, we found that the European crabapple, $M$. sylvestris, likely had a greater genomic contribution to cider apples than to dessert apples. We examined clonal and first-degree relationships, finding widespread use of a few commercially successful cultivars during apple breeding. The use of a small number of 'elite' cultivars in apple fails to exploit the immense 
genomic and phenomic diversity available and leaves the apple industry vulnerable to evolving pests and pathogens and a changing climate. Lastly, we identified evidence of selection for red and firm apples during apple improvement. Ultimately, germplasm collections such as the USDA apple collection described in this study will serve as an essential source of diverse accessions and wild relatives, which have enormous potential for future plant improvement.

\section{Reporting summary}

Further information on research design is available in the Nature Research Reporting Summary linked to this article.

\section{Acknowledgements}

This research was supported in part by funding from the National Sciences and Engineering Research Council of Canada and Canada Research Chairs programme (SM). Z.M. was supported by National Science Foundation Plant Genome Research Programme 1546869. We thank Gavin Douglas for bioinformatic support of this project. We gratefully acknowledge everyone who established and maintains the USDA apple germplasm collection in Geneva, NY.

\section{Author details}

${ }^{1}$ Department of Plant, Food, and Environmental Sciences,

Faculty of Agriculture, Dalhousie University, Truro, NS, Canada. ${ }^{2}$ USDA-ARS, Plant Germplasm Preservation Research Unit, Fort Collins, CO, USA. ${ }^{3}$ USDA-ARS, Grape Genetics Research Unit, Geneva, NY, USA. ${ }^{4}$ Present address: Agriculture and Agri-Food Canada, Fredericton Research and Development Centre, Fredericton, NB, Canada

\section{Author contributions}

G.-Y.Z. and S.M. designed the study; Z.M. and K.M.G. performed analyses; C.R., C. T.C., H.R.S., and G.F. performed data acquisition and interpretation; Z.M., K.M.G., and S.M. drafted the manuscript and all authors helped revise it.

\section{Conflict of interest}

The authors declare that they have no conflict of interest.

Supplementary Information accompanies this paper at (https://doi.org/ 10.1038/s41438-020-00441-7).

Received: 5 October 2020 Accepted: 9 November 2020 Published online: 01 January 2021

\section{References}

1. Miller, A. J. \& Gross, B. L. From forest to field: perennial fruit crop domestication. Am. J. Bot. 98, 1389-414 (2011).

2. Food and Agriculture Organization of the United Nations. FAOSTAT. http:// faostat3.fao.org/browse/rankings/commodities by regions/E (2016).

3. Vavilov, N. I. Pyat'Kontinentov [Five Continents]. (Leningrad: Nauka, 1987).

4. Velasco, R. et al. The genome of the domesticated apple (Malus $x$ domestica Borkh.). Nat. Genet. 42, 833-9 (2010).

5. Duan, N. et al. Genome re-sequencing reveals the history of apple and supports a two-stage model for fruit enlargement. Nat. Commun. 8, 249, https://doi.org/10.1038/s41467-017-00336-7 (2017).

6. Cornille, A. et al. New insight into the history of domesticated apple: secondary contribution of the European wild apple to the genome of cultivated varieties. PLoS Genet. 8, e1002703 (2012).

7. Gross, B. L., Henk, A. D., Richards, C. M., Fazio, G. \& Volk, G. M. Genetic diversity in Malus xdomestica (Rosaceae) through time in response to domestication. Am. J. Bot. 101, 1770-9 (2014).

8. World Apple and Pear Association. European Apple and Pear Crop Forecast. http://www.wapa-association.org/asp/page_1.asp?doc_id=447 (2018).
9. Proctor, J. The 'McIntosh' apple. Fruit. Var. J. 44, 50-53 (1990).

10. U.S. Apple Association. Apple Industry At-a-Glance. http://usapple.org/theindustry/apple-industry-at-a-glance/ (2018).

11. Elshire, R. J. et al. A robust, simple genotyping-by-sequencing (GBS) approach for high diversity species. PLOS ONE 6, e19379 (2011).

12. Daccord, N. et al. High-quality de novo assembly of the apple genome and methylome dynamics of early fruit development. Nat. Genet. https:/doi.org/ 10.1038/ng.3886 (2017)

13. Li, H. \& Durbin, R. Fast and accurate short read alignment with BurrowsWheeler transform. Bioinformatics 25, 1754-60 (2009).

14. Glaubitz, J. C. et al. TASSEL-GBS: a high capacity genotyping by sequencing analysis pipeline. PLOS ONE 9, e90346 (2014).

15. Danecek, P. et al. The variant call format and VCFtools. Bioinformatics 27, 2156-8 (2011).

16. Money, D., Migicovsky, Z., Gardner, K. \& Myles, S. LinklmputeR: user-quided genotype calling and imputation for non-model organisms. BMC Genomics 18, 523 (2017).

17. Purcell, S. PLINK v.1.07. http://pngu.mgh.harvard.edu/purcell/plink/ (2009).

18. Purcell, S. et al. PLINK: a tool set for whole-genome association and population-based linkage analyses. Am. J. Hum. Genet. 81, 559-575 (2007).

19. Larsen, B. et al. Population structure, relatedness and ploidy levels in an apple gene bank revealed through genotyping-by-sequencing. PLOS ONE 13, e0201889 (2018).

20. Raj, A., Stephens, M. \& Pritchard, J. K. fastSTRUCTURE: variational inference of population structure in large SNP data sets. Genetics 197, 573-89 (2014).

21. Patterson, N., Price, A. L. \& Reich, D. Population structure and eigenanalysis. PLoS Genet. 2, e190 (2006).

22. McVean, G. A genealogical interpretation of principal components analysis. PLoS Genet. 5, e1000686 (2009).

23. Migicovsky, Z. et al. Genomic ancestry estimation quantifies use of wild species in grape breeding. BMC Genomics 17, 478 (2016).

24. Sawler, J. et al. Genomics assisted ancestry deconvolution in grape. PLOS ONE 8, e80791 (2013).

25. Migicovsky, Z. et al. Genome to phenome mapping in apple using historical data. Plant Genome 9 https://doi.org/10.3835/plantgenome2015.11.0113 (2016).

26. Patterson, N. et al. Ancient admixture in human history. Genetics 192, 1065-93 (2012).

27. Weir, B. S. \& Cockerham, C. C. Estimating F-statistics for the analysis of population structure. Evolution 38, 1358-1370 (1984).

28. Scheet, P. \& Stephens, M. A fast and flexible statistical model for large-scale population genotype data: applications to inferring missing genotypes and haplotypic phase. Am. J. Hum. Genet. 78, 629-644 (2006).

29. Szpiech, Z. A. \& Hernandez, R. D. selscan: an efficient multithreaded program to perform EHH-based scans for positive selection. Mol. Biol. Evol. 31, 2824-7 (2014).

30. Jung, S. et al. 15 years of GDR: New data and functionality in the Genome Database for Rosaceae. Nucleic Acids Res. https://doi.org/10.1093/nar/gky1000 (2018).

31. Alexa, A. \& Rahnenfuhrer, J. topGO: Enrichment Analysis for Gene Ontology. (R package version 2.28.0, 2016)

32. Butts, C. T. network: a package for managing relational data in R. J. Stat. Softw. 24, 1-36 (2008)

33. Noiton, D. A. \& Alspach, P. A. Founding clones, inbreeding, coancestry, and status number of modern apple cultivars. J. Am. Soc. Hortic. Sci. 121, 773-782 (1996).

34. Hokanson, S., Szewc-McFadden, A., Lamboy, W. \& McFerson, J. Microsatellite (SSR) markers reveal genetic identities, genetic diversity and relationships in a Malus $\times$ domestica Borkh. core subset collection. Theor. Appl. Genet. 97, 671-683 (1998)

35. Bradbury, P. J. et al. TASSEL: software for association mapping of complex traits in diverse samples. Bioinformatics 23, 2633-2635 (2007).

36. Segura, V. et al. An efficient multi-locus mixed-model approach for genomewide association studies in structured populations. Nat. Genet. 44, 825-30 (2012)

37. Turner, S. D. qqman: an R package for visualizing GWAS results using Q-Q and manhattan plots. J. Open Source Softw. 3, 731, https://doi.org/10.21105/ joss.00731 (2018).

38. Takos, A. M. et al. Light-induced expression of a MYB gene regulates anthocyanin biosynthesis in red apples. Plant Physiol. 142, 1216-32 (2006).

39. Espley, R. V. et al. Red colouration in apple fruit is due to the activity of the MYB transcription factor, MdMYB10. Plant J. 49, 414-27 (2007).

40. Ban, Y. et al. Isolation and functional analysis of a MYB transcription factor gene that is a key regulator for the development of red coloration in apple skin. Plant Cell Physiol. 48, 958-70 (2007). 
41. Chagné, D. et al. A functional genetic marker for apple red skin coloration across different environments. Tree Genet. Genomes 12, 67 (2016).

42. Zhang, L. et al. A high-quality apple genome assembly reveals the association of a retrotransposon and red fruit colour. Nat. Commun. 10, 1494 (2019).

43. Larsen, B. et al. Genome-wide association studies in apple reveal loci for aroma volatiles, sugar composition, and harvest date. Plant Genome 12 (2019).

44. Yeats, T. H. et al. Allelic diversity of NAC18.1 is a major determinant of fruit firmness and harvest date in apple (Malus domestica). https://www.biorxiv. org/content/10.1101/708040v1 (in review).

45. Kumar, S., Raulier, P., Chagné, D. \& Whitworth, C. Molecular-level and trait-level differentiation between the cultivated apple (Malus $\times$ domestica Borkh.) and its main progenitor Malus sieversii. Plant Genet. Resour. 12, 330-340 (2014).

46. Harris, S. A., Robinson, J. P. \& Juniper, B. E. Genetic clues to the origin of the apple. Trends Genet. 18, 426-430 (2002).

47. Cornille, A., Giraud, T., Smulders, M. J. M., Roldán-Ruiz, I. \& Gladieux, P. The domestication and evolutionary ecology of apples. Trends Genet. 30, 57-65 (2014).

48. Volk, G. M. et al. Chloroplast heterogeneity and historical admixture within the genus Malus. Am. J. Bot. 102, 1198-1208 (2015).

49. Cornille, A. et al. A multifaceted overview of apple tree domestication. Trends Plant Sci. (2019) https://doi.org/10.1016/j.tplants.2019.05.007.

50. Sun, $X$. et al. Phased diploid genome assemblies and pan-genomes provide insights into the genetic history of apple domestication. Nat. Genet. 52, 1423-1432, https://doi.org/10.1038/s41588-020-00723-9 (2020).

51. Myles, S. et al. Genetic structure and domestication history of the grape. Proc. Natl Acad. Sci. 108, 3530-3535 (2011)

52. Dobrenel, T. et al. Regulation of plant growth and metabolism by the TOR kinase. Biochem. Soc. Trans. 39, 477-481 (2011).

53. Kelsey, D. \& Brown, S. 'Mclntosh Wijcik': a columnar mutation of 'McIntosh' apple proving useful in physiology and breeding research. Fruit Var. J. 46, 83-87 (1992).

54. Luby, J. J. \& Bedford, D. S. Cultivars as consumer brands: trends in protecting and commercializing apple cultivars via intellectual property rights. Crop Sci. 55, 2504-2510 (2015).

55. Komatsu, H. Red Fuji in Japan: choosing the best strain for your business strategy. Compact Fruit Tree 31, 44-45 (1998).

56. Roach, M. J. et al. Population sequencing reveals clonal diversity and ancestral inbreeding in the grapevine cultivar Chardonnay. PLoS Genet. 14, e1007807 (2018).

57. Ballard, J. K. Granny Smith: an Important Apple for the Pacific Northwest (Pullman, 1981).
58. Muranty, $\mathrm{H}$. et al. Using whole-genome SNP data to reconstruct a large multi-generation pedigree in apple germplasm. BMC Plant Biol. 20, 2 (2020).

59. Warschefsky, E. J. \& von Wettberg, E. J. B. Population genomic analysis of mango (Mangifera indica) suggests a complex history of domestication. N. Phytol. 222, 2023-2037 (2019).

60. Cabe, P. R., Baumgarten, A., Onan, K., Luby, J. J. \& Bedford, D. S. Using microsatellite analysis to verify breeding records: a study of 'honeycrisp' and other cold-hardy apple cultivars. Hortscience 40, 15-17 (2005).

61. Howard, N. P. et al. Elucidation of the 'Honeycrisp' pedigree through haplotype analysis with a multi-family integrated SNP linkage map and a large apple (Malus x domestica) pedigree-connected SNP data set. Hortic. Res. 4, 17003 (2017).

62. Migicovsky, Z. \& Myles, S. Exploiting wild relatives for genomics-assisted breeding of perennial crops. Front. Plant Sci. 460 (2017)..

63. Valois, S., Merwin, I. A. \& Padilla-Zakour, O. I. Characterization of fermented cider apple varieties grown in upstate New York. J. Am. Pomol. Soc. 60, 113-128 (2006).

64. Sanoner, P., Guyot, S., Marnet, N., Molle, D. \& Drilleau, J.-F. Polyphenol profiles of French cider apple varieties (Malus domestica sp.). J. Agric. Food Chem. 47, 4847-4853 (1999).

65. Hamadziripi, E. T., Theron, K. I., Muller, M. \& Steyn, W. J. Apple compositional and peel color differences resulting from canopy microclimate affect consumer preference for eating quality and appearance. HortScience 49, 384-392 (2014).

66. Hazzouri, K. M. et al. Genome-wide association mapping of date palm fruit traits. Nat. Commun. 10, 4680 (2019).

67. Huang, D. et al. Subfunctionalization of the Ruby2-Ruby1 gene cluster during the domestication of citrus. Nat. Plants 4, 930-941 (2018).

68. Legun, K. Ever-redder apples: How aesthetics shape the biology of markets. In Biological Economies: Experimentation and the politics of agri-food frontiers (eds Heron, R. L., Campbell, H., Lewis, N., \& Carolan, M.) 127-140 (Routledge, 2016).

69. Hampson, C. R. \& Kemp, H. in Apples: Botany, Production and Uses (eds. Ferree, D. C. \& Warrington, I. J.) 61-89 (CABI Publishing, 2003).

70. Laurens, F. et al. An integrated approach for increasing breeding efficiency in apple and peach in Europe. Hortic. Res. 5, 11 (2018).

71. $\mathrm{Wu}, \mathrm{J}$. et al. Diversification and independent domestication of Asian and European pears. Genome Biol. 19, 77 (2018).

72. Urrestarazu, J. et al. Genome-wide association mapping of flowering and ripening periods in apple. Front. Plant Sci. 8, 1923 (2017).

73. McClure, K. A. et al. A genome-wide association study of apple quality and scab resistance. Plant Genome 11, 170075 (2018). 